PROCEEDINGS OF THE

AMERICAN MATHEMATICAL SOCIETY

Volume 127, Number 1, January 1999, Pages 79-87

S 0002-9939(99)04998-9

\title{
TWO-WEIGHTED ESTIMATIONS FOR THE HARDY-LITTLEWOOD MAXIMAL FUNCTION IN IDEAL BANACH SPACES
}

\author{
E. I. BEREZHNOI
}

(Communicated by J. Marshall Ash)

\begin{abstract}
We give conditions on a couple of ideal Banach spaces with weights which are both necessary and sufficient for the Hardy-Littlewood maximal function to satisfy the two-weighted estimations of weak type, and we consider a modification of the Hardy-Littlewood maximal function. We also give some conditions on weights in order for the Hardy-Littlewood maximal function and the modification under consideration to fulfil the two-weighted estimations of strong type.
\end{abstract}

\section{INTRODUCTION}

The general problem of two-weighted estimations of weak type in $L_{p}$-spaces for the Hardy-Littlewood maximal function has been solved in [9]. In the case of Lorentz spaces $L_{p, q}$ a corresponding result has been obtained in $[2,7]$ (see also [3, 4]). In order to obtain a positive answer the $A_{p}$-condition for weights is required in both cases mentioned. The problem of two-weighted estimations of strong type for the Hardy-Littlewood maximal function in $L_{p}$-spaces has been considered in [12]. The solution is boundedness of the operator of the Hardy-Littlewood maximal function on the set of the characteristic functions of cubes. Simpler conditions, necessary and sufficient on a certain class of weights, have been considered by many authors (see for example [3] or [4]). Among recent papers we should mention [11], where, for a large class of weights, there are given necessary and sufficient conditions for boundedness of the weighted fractional maximal operator.

In the present paper we give necessary and sufficient conditions for two-weighed estimations of weak type for the Hardy-Littlewood maximal function and a modification in ideal Banach spaces. We also give some conditions on weights assuring two-weighted estimations of strong type for the Hardy-Littlewood maximal function and a modification. The obtained results are new even in the case of Orlicz spaces.

\section{Preliminaries}

Let $S=S\left(\mathbf{R}^{n}, \mu\right)$ be the space of all Lebesgue measurable real-valued functions on $\mathbf{R}^{n}$ endowed with the topology of convergence in measure on each set of finite measure. Recall that a Banach subspace $X$ in $S$ is said to be ideal [8] if $x \in X$,

Received by the editors December 11, 1991 and, in revised form, September 19, 1996.

1991 Mathematics Subject Classification. Primary 42B20, 42B25.

(C)1999 American Mathematical Society 
$y \in S$ and $|y| \leq|x|$ a.e. imply $\|y\| \leq\|x\|$. Classical examples of ideal Banach spaces are $L_{p}$-spaces, Lorentz spaces $L_{p, q}$, Orlicz spaces $L_{h}$, and symmetric spaces [8].

By $X^{\prime}$ we denote the ideal Banach space dual (in the sense of Köthe) to $X$, which can be identified with the space of all functionals possessing an integral representation, that is,

$$
X^{\prime}=\left\{y=S:\|y\|_{X^{\prime}}=\sup \left\{\int_{\mathbf{R}^{n}}|x y| d \mu:\|x\|_{X} \leq 1\right\}<\infty\right\} .
$$

An ideal Banach space $X$ is said to be minimal if the closed linear span $\left\{\chi_{A}: \mu(A)\right.$ $<\infty\}$ is dense in $X$, where $\chi_{A}$ stands for the characteristic function of the set $A$. In what follows we suppose that all ideal Banach spaces have the Fatou property [8], i.e. the unit balls of these spaces are closed subsets in $S$. Thus if $X$ is an ideal Banach space, then $X=X^{\prime \prime}$ with equality of the norms.

Let $X$ be an ideal Banach space and let $w$ be a weight, i.e. a positive measurable function on $S$. By $X_{w}$ we shall denote the ideal Banach space $\{x \in S: x w \in X\}$ equipped with the norm $\|x\|_{X_{w}}=\|x w\|_{X}$. It is easily seen that

$$
\left(X_{w}\right)^{\prime}=X_{w^{-1}}^{\prime} \text {. }
$$

If $T: X \rightarrow Y$ is a bounded operator between Banach spaces $X$ and $Y$, then we shall say that $T$ is of strong type (between $X$ and $Y$ ).

Definition 1. An operator $T: X \rightarrow S$ is said to be of weak type $(X, Y)$ if there exists a constant $C>0$ such that

$$
\left\|\chi_{D(\lambda, T x)}\right\|_{Y} \leq C \lambda^{-1}\|x\|_{X}
$$

for any $x \in X$ and $\lambda>0$, where $D(\lambda, f)=\left\{t \in \mathbf{R}^{n}:|f(t)|>\lambda\right\}$.

Note that if $X=L_{p}, Y=L_{q}$, then (2) is the classical inequality of weak type (see $[3,4])$. Since $Y=Y^{\prime \prime}$ and the inequality

$$
\int_{\mathbf{R}^{n}}|y T x| d \mu \geq \int_{\{t:|T x|>\lambda\}} \lambda|y| d \mu=\lambda \int_{\mathbf{R}^{n}}\left|y \chi_{D(\lambda, T x)}\right| d u
$$

holds for $x \in X$ and $y \in Y^{\prime}$, it follows that $T$ is of weak type, whenever $T$ is of strong type.

Let $\mathbf{B}$ be a family of cubes in $\mathbf{R}^{n}$ with sides parallel to the coordinate axes, and let $\varphi: \mathbf{R}_{+} \rightarrow \mathbf{R}_{+}$be an increasing function. Then the Hardy-Littlewood maximal function $M_{\varphi}$ relative to $\mathbf{B}$ and $\varphi$ is defined on the space of locally integrable functions on $\mathbf{R}^{n}$ by the formula

$$
M_{\varphi} x(t)=\sup \left\{\frac{1}{\varphi(|Q|)} \int_{Q}|x| d \mu: Q \in \mathbf{B}, t \in Q\right\},
$$

where $|Q|$ denotes the Lebesgue measure of $Q$.

The main problem we are interested in is the following: Let $X, Y$ be ideal Banach spaces and $w, v$ some weights. Under which conditions on $X, Y$ and $\varphi$ is the operator $M_{\varphi}$ of weak (resp. of strong) type $\left(X_{w}, Y_{v}\right)$ ?

Let $\bar{Q}=\left\{Q_{i}\right\}$ be a subfamily of $\mathbf{B}$ with $Q_{i}^{0} \cap Q_{j}^{0}=\varnothing$ for $i \neq j$, where $Q^{0}$ denotes the interior of a cube $Q$. The averaging operator relative to $\bar{Q}$ and $\varphi$ is defined by

$$
T_{\bar{Q}} x=\sum_{i}\left(\frac{1}{\varphi\left(\left|Q_{i}\right|\right)} \int_{Q_{i}} x d \mu\right) \chi_{Q_{i}}
$$


for $x \in L_{1}^{\text {loc }}$. If $\bar{Q}$ contains only one cube $Q$, then the corresponding operator is denoted by $T_{Q}$.

Let $X$ and $Y$ be ideal Banach spaces. We need the following

Definition 2. A pair of weights $(w, v)$ is said to be a member of the class $A_{\varphi}(X, Y)$ $\left((w, v) \in A_{\varphi}(X, Y)\right)$ if

$$
C_{0}(\varphi, w, v)=\sup _{Q \in \mathbf{B}} \frac{1}{\varphi(|Q|)}\left\|v \chi_{Q}\right\|_{Y}\left\|w^{-1} \chi_{Q}\right\|_{X^{\prime}}<\infty .
$$

Note that if $X=Y=L_{p}, \varphi(t)=t$ for $t>0$, then (3) is simply the Muckenhoupt $A_{p^{-}}$condition $[3,4]$.

\section{Results}

The following simple result shows that the condition (3) is equivalent to the uniform boundedness of the norms of $T_{Q}: X_{w} \rightarrow Y_{v}$.

Lemma 2.1 (see also $[6]) .(w, v) \in A_{\varphi}(X, Y)$ if and only if

$$
C=\sup \left\{\left\|T_{Q}\right\|_{X_{w} \rightarrow Y_{v}}: Q \in \mathbf{B}\right\}<\infty ;
$$

moreover $C=C_{0}(\varphi, w, v)$.

Definition 3. A pair of ideal Banach spaces is said to have the property $\mathbf{G}(\mathbf{B})$ $((X, Y) \in \mathbf{G}(\mathbf{B}))$ if there exists a constant $C_{1}=C_{1}(\mathbf{B}, X, Y)$ such that

$$
\sum_{i}\left\|x \chi_{Q_{i}}\right\|_{X}\left\|y \chi_{Q_{i}}\right\|_{Y^{\prime}} \leq C_{1}\|x\|_{X}\|y\|_{Y^{\prime}}
$$

for any family $\left\{Q_{i}: Q_{i} \in \mathbf{B}\right\}$ of disjoint cubes and every $x \in X, y \in Y^{\prime}$.

If we can take any disjoint measurable sets instead of $\mathbf{B}$ and cubes in the inequality (4), then we shall write $(X, Y) \in \mathbf{G}$.

Let us remark that $\left(L_{p}, L_{q}\right) \in \mathbf{G}$ if and only if $p \leq q$.

Theorem 2.1. Assume that $(w, v)$ is a pair of weights and that $(X, Y)$ is a pair of ideal Banach spaces with $(X, Y) \in \mathbf{G}(\mathbf{B})$. Then

$$
\sup _{\bar{Q}}\left\|T_{\bar{Q}}\right\|_{X_{w} \rightarrow Y_{v}}<\infty
$$

if and only if $(w, v) \in A_{\varphi}(X, Y)$.

Proof. Necessity follows from Lemma 2.1. In order to prove sufficiency take any $x \in X_{w}$ and $y \in\left(Y_{v}\right)^{\prime}$. Then, by (1) it follows that

$$
\int y T_{\bar{Q}} x d \mu=\sum_{i} \frac{1}{\varphi\left(\left|Q_{i}\right|\right)} \int_{Q_{i}}\left(y v^{-1} / v^{-1}\right) d \mu \int_{Q_{i}}(x w / w) d \mu .
$$

Duality and the condition $A_{\varphi}(X, Y)$ yield

$$
\frac{1}{\varphi(|Q|)}\left(\int_{Q} g v d \mu\right)\left(\int_{Q}(f / w) d \mu\right) \leq C_{0}(\varphi, w, v)\left\|g \chi_{Q}\right\|_{Y^{\prime}}\left\|f \chi_{Q}\right\|_{X}
$$

for any $f \in X$ and $g \in Y^{\prime}$. Hence

$$
\int y T_{\bar{Q}} x d \mu \leq C_{0}(\varphi, w, v) \sum_{i}\left\|x w \chi_{Q_{i}}\right\|_{X}\left\|y v^{-1} \chi_{Q_{i}}\right\|_{Y^{\prime}}
$$


Since $(X, Y) \in \mathbf{G}(\mathbf{B})$,

$$
\int y T_{\bar{Q}} x d \mu \leq C_{0}(\varphi, w, v) C_{1}(\mathbf{B}, X, Y)\|x\|_{X_{w}}\|y\|_{\left(Y_{v}\right)^{\prime}} .
$$

This implies that

$$
\left\|T_{\bar{Q}}\right\|_{X_{w} \rightarrow Y_{v}} \leq C_{0}(\varphi, w, v) C_{1}(\mathbf{B}, X, Y)
$$

and the proof is finished.

In the proof of the above theorem the condition $(X, Y) \in \mathbf{G}(\mathbf{B})$ was used. Now we give simple sufficient conditions on $X$ and $Y$ assuring $(X, Y) \in \mathbf{G}(\mathbf{B})$.

Following [8], a Banach space $X$ is said to satisfy an upper p-estimate, respectively, lower $p$-estimate, for some $p \in(1, \infty)$ if there exists a constant $C<\infty$ such that every finite sequence $\left\{x_{i}\right\}_{i=1}^{n}$ of disjointly supported elements in $X$ satisfies

$$
\left\|\sum_{i=1}^{n} x_{i}\right\| \leq C\left(\sum_{i=1}^{n}\left\|x_{i}\right\|^{p}\right)^{1 / p},
$$

respectively,

$$
\left(\sum_{i=1}^{n}\left\|x_{i}\right\|^{p}\right)^{1 / p} \leq C\left\|\sum_{i=1}^{n} x_{i}\right\| .
$$

Theorem 2.2. Suppose $X$ satisfies a lower $p$-estimate and $Y$ satisfies an upper $q$-estimate with $p \leq q$. Then $(X, Y) \in \mathbf{G}$.

Proof. If $Y$ satisfies an upper $q$-estimate, then by duality (see [8]), it follows that $Y^{\prime}$ satisfies a lower $q^{\prime}$-estimate, where, as usual, $1 / q+1 / q^{\prime}=1$. Since $p \leq q, 1 / p+1 / q^{\prime} \geq$ 1. Thus, applying Hölder's inequality, we obtain the desired assertion.

For the classical Orlicz and Lorentz spaces the values of the upper and lower estimates are well known [5, 10]. For the Lorentz spaces $L_{p, q}$ a corresponding result may be easily obtained by Lemma 2.5 in [2]. Different conditions for inclusion $(X, Y) \in \mathbf{G}$ can be found in [1]. Here $(X, Y)$ is a pair of symmetric spaces (Lebesgue, Orlicz, Lorentz, Marcinkiewicz).

Theorem 2.3. Let $(X, Y)$ be a pair of ideal Banach spaces with $(X, Y) \in \mathbf{G}(\mathbf{B})$. Then the operator $M_{\varphi}$ is of weak type $\left(X_{w}, Y_{v}\right)$ if and only if $(w, v) \in A_{\varphi}(X, Y)$.

Proof. Since $M_{\varphi} x \geq T_{Q} x$ a.e. for any $x \in X_{w}$, the necessity of the condition $(w, v) \in A_{\varphi}(X, Y)$ is obvious.

Now suppose $(w, v) \in A_{\varphi}(X, Y)$. Take any $x \in X_{w}, x \geq 0$ a.e., and put $y=M_{\varphi} x$. Then applying the theorem of Besikhovic $[2,3]$ we can assume that

$$
\{t: y(t)>\lambda\} \subseteq \bigcup Q_{j i},
$$

where the first index takes the values $j=1,2, \ldots, 2^{n} ; Q_{j k}^{0} \cap Q_{j i}^{0}=\varnothing$ for $i \neq k$ and

$$
\frac{1}{\varphi\left(\left|Q_{j i}\right|\right)} \int_{Q_{j i}} x d \mu>\lambda
$$

It is not difficult to see that in order to show that $M_{\varphi}$ is of weak type $\left(X_{w}, Y_{v}\right)$ it suffices to prove the uniform boundedness of the norms of the averaging operators 
$T_{j}: X_{w} \rightarrow Y_{v}$,

$$
T_{j} x=\sum_{i} \frac{1}{\varphi\left(\left|Q_{j i}\right|\right)}\left(\int_{Q_{j i}} x d \mu\right) \chi_{Q_{j i}}, \quad j=1,2, \ldots, 2^{n} .
$$

But this holds by Theorem 2.1, and the proof is finished.

In order to prove the boundedness of the Hardy-Littlewood maximal function $M_{\varphi}$ between $X_{w}$ and $Y_{v}$ some additional assumptions are needed.

We assume that $\mathbf{B}$ contains dyadic cubes, of side-length bounded by the number $2^{k_{0}}$ ( $k_{0}$ is fixed), i.e. $Q=\prod_{i=1}^{n}\left[x_{i}, x_{i}+2^{k}\right]$, where $x \in 2^{k} \mathbf{Z}$ and $k \leq k_{0}$.

Definition 4. A family of dyadic cubes is said to be maximal if the interiors of any two different cubes do not intersect each other.

Now let $\left\{Q_{i}: i \in I\right\}$ be a certain family of cubes in B. By $\left\{Q_{i}: i \in \bar{I}\right\}$ we denote a maximal subfamily of $\left\{Q_{i}: i \in I\right\}$ such that every cube of $\left\{Q_{i}: i \in \bar{I}\right\}$ is not contained in any other cube of the family $\left\{Q_{i}: i \in I\right\}$. Since the side-length of dyadic cubes is bounded by $2^{k_{0}}$, we have

$$
\begin{gathered}
Q_{k}^{0} \cap Q_{j}^{0}=\varnothing \quad(k, j \in \bar{I} ; k \neq j), \\
\bigcup\left\{Q_{k}: k \in I\right\}=\bigcup\left\{Q_{k}: k \in \bar{I}\right\} .
\end{gathered}
$$

It is obvious that the method of construction of the family $\left\{Q_{i}: i \in \bar{I}\right\}$, by using $\left\{Q_{i}: i \in I\right\}$, is the method of construction of the maximal cubes from $\left\{Q_{i}: i \in I\right\}$.

In the sequel we suppose that the increasing function $\varphi: \mathbf{R}_{+} \rightarrow \mathbf{R}_{+}$satisfies the following conditions:

$$
\varphi\left(\sum_{i} t_{i}\right) \leq C \sum_{i} \varphi\left(t_{i}\right), \quad t_{i} \in \mathbf{R}_{+}
$$

there exist constants $\gamma, \alpha \in(0,1)$ such that for $t_{1}, t_{2} \in \mathbf{R}_{+}$

$$
\frac{\varphi\left(t_{1}\right)}{\varphi\left(t_{2}\right)} \leq \gamma \quad \text { implies } \quad \frac{t_{1}}{t_{2}} \leq \alpha .
$$

In fact (8) implies (9). In order to prove this let $\rho(s)=\sup \left\{\varphi(s t) / \varphi(t): t \in \mathbf{R}_{+}\right\}$. Clearly $\rho$ is increasing and submultiplicative, i.e. $\rho(s t) \leq \rho(s) \rho(t)$ for all $s, t>0$. Thus, by (8) it follows that $\rho(2) \leq 2 c$. Hence $\rho(s)<\infty$ for all $s>0$. Let $K=\sup \{\rho(s): s \leq 2\}$ and $\gamma=\frac{1}{2 K}$. We claim that (9) is true with $\alpha=1 / 2$. Indeed, if $t_{1}=s t_{2}$ and $\varphi\left(s t_{2}\right) \leq \gamma \varphi\left(t_{2}\right)$, then $\rho\left(s^{-1}\right) \geq \gamma^{-1}=2 K$. This yields $s^{-1}>2$ and $s<0.5$.

Let us remark that quasi-concave functions satisfy the condition (8) and thus (9).

In what follows $M$ denotes the classical Hardy-Littlewood maximal function, i.e. $M=M_{\varphi}$ with $\varphi(t)=t$ for $t>0$.

Theorem 2.4. Let two minimal ideal Banach spaces $X, Y$ satisfy $(X, Y) \in \mathbf{G}$. If the operator $M$ is bounded from $X_{w}$ into $X_{w}$, then $M_{\varphi}$ is of strong type $\left(X_{w}, Y_{v}\right)$ if and only if $(w, v) \in A_{\varphi}(X, Y)$ and $\left\|M_{\varphi}\right\|_{X_{w} \rightarrow Y_{v}}$ does not depend on $k_{0}$.

Proof. The necessity of the condition $(w, v) \in A_{\varphi}(X, Y)$ is obvious. In order to prove sufficiency let us point out that we shall use operators with infinitely many components. The minimality of $X$ and $Y$ allow us to consider only a finite number 
and then to prove the uniform boundedness of the norms of the restricted operators. This remark will often be used below. Take $t \in \mathbf{R}_{+}$such that the following holds:

$$
\gamma>C \frac{\rho\left(2^{n}\right)}{t}
$$

with $C$ from (8).

Now let $x \in X_{w}, x \geq 0$ a.e. We put

$$
\Omega_{k}=\left\{s: M_{\varphi} x>t^{k}, k \in \mathbf{Z}\right\} .
$$

Each $\Omega_{k}$ is the union of certain cubes from $\mathbf{B}$, i.e.

$$
\Omega_{k}=\left\{Q_{k i}: i \in I_{k}\right\} .
$$

For each $k \in \mathbf{Z}$ in $\left\{Q_{k i}: i \in I_{k}\right\}$ we choose a maximal subfamily, by the method described above, and denote it by $\left\{Q_{k i}: i \in \bar{I}_{k}\right\}$.

For each $Q_{k i}, i \in \bar{I}_{k}$, let $E_{k i}=Q_{k i} \backslash \Omega_{k+1}$. We claim that

$$
\left|E_{k i}\right|>(1-\alpha)\left|Q_{k i}\right|,
$$

where $\alpha$ is as in (9). Indeed, by the maximality of $\left\{Q_{k i}\right\},(8)$ and the structure of $\Omega_{k+1}$, it follows that

$$
\begin{aligned}
t^{k} \varphi\left(2^{n}\left|Q_{k i}\right|\right) & \geq \int_{Q_{k i}} x d \mu \\
& \geq\left\{\sum \frac{\varphi\left(\left|Q_{k+1 j}\right|\right)}{\varphi\left(\left|Q_{k+1 j}\right|\right)} \int_{Q_{k+1 j}} x d \mu: Q_{k+1 j} \subset Q_{k i}, j \in \overline{I_{k+1}}\right\} \\
& \geq t^{k+1}\left\{\sum \varphi\left(\left|Q_{k+1 j}\right|\right): Q_{k+1 j} \subset Q_{k i}, j \in \overline{I_{k+1}}\right\} \\
& \geq C^{-1} t^{k+1}\left\{\varphi\left(\sum\left|Q_{k+1 j}\right|\right): Q_{k+1 j} \subset Q_{k i}, j \in \overline{I_{k+1}}\right\} .
\end{aligned}
$$

Hence

$$
\varphi\left(2^{n}\left|Q_{k i}\right|\right) \geq C^{-1} t \varphi\left(\left|Q_{k i} \cap \Omega_{k+1}\right|\right) .
$$

This simply yields

$$
C t^{-1} \rho\left(2^{n}\right) \geq \frac{\varphi\left(\left|Q_{k i} \cap \Omega_{k+1}\right|\right)}{\varphi\left(\left|Q_{k i}\right|\right)} .
$$

(11) follows from (9) and (10).

Now we define three operators

$$
\begin{aligned}
& T_{0} y=\sum_{k \in \mathbf{Z}, j \in \overline{I_{k}}}\left(\frac{1}{\varphi\left(\left|Q_{k j}\right|\right)} \int_{Q_{k j}} y d \mu\right) \chi_{E_{k j}}, \\
& T_{1} y=\sum_{k \in \mathbf{Z}, j \in \overline{I_{k}}}\left(\frac{1}{\left|Q_{k j}\right|} \int_{Q_{k j}} y d \mu\right) \chi_{E_{k j}}, \\
& T_{2} y=\sum_{k \in \mathbf{Z}, j \in \overline{I_{k}}}\left(\frac{1}{\varphi\left(\left|Q_{k j}\right|\right)} \int_{E_{k j}} y d \mu\right) \chi_{E_{k j}},
\end{aligned}
$$


and their duals

$$
\begin{aligned}
T_{0}^{\prime} f & =\sum_{k \in \mathbf{Z}, j \in \overline{I_{k}}}\left(\frac{1}{\varphi\left(\left|Q_{k j}\right|\right)} \int_{E_{k j}} f d \mu\right) \chi_{Q_{k j}}, \\
T_{1}^{\prime} f & =\sum_{k \in \mathbf{Z}, j \in \overline{I_{k}}}\left(\frac{1}{\left(\left|Q_{k j}\right|\right)} \int_{E_{k j}} f d \mu\right) \chi_{Q_{k j}}, \\
T_{2}^{\prime} & =T_{2} .
\end{aligned}
$$

We have

$$
\begin{aligned}
\left\|M_{\varphi} x\right\|_{Y_{v}} & \leq\left\|\sum_{k} t^{k+1} \chi_{\Omega_{k} \backslash \Omega_{k+1}}\right\|_{Y_{v}} \\
& \leq t\left\|\sum_{k \in \mathbf{Z}, j \in \overline{I_{k}}}\left(\frac{1}{\varphi\left(\left|Q_{k j}\right|\right)} \int_{Q_{k j}} x d \mu\right) \chi_{E_{k j}}\right\|_{Y_{v}}=t\left\|T_{0} x\right\|_{Y_{v}} .
\end{aligned}
$$

In order to finish the proof it suffices to show that

$$
\left\|T_{0}^{\prime}\right\|_{\left(Y_{v}\right)^{\prime} \rightarrow\left(X_{w}\right)^{\prime}}<\infty .
$$

Let $f \geq 0$ a.e. Then, using (11), we have

$$
\begin{aligned}
T_{0}^{\prime} f & =\sum_{k \in \mathbf{Z}, j \in \overline{I_{k}}}\left(\frac{1}{\varphi\left(\left|Q_{k j}\right|\right)} \int_{E_{k j}} f d \mu\right) \chi_{Q_{k j}} \\
& =\sum_{k \in \mathbf{Z}, j \in \overline{I_{k}}}\left(\frac{\left|Q_{k j}\right|}{\left|E_{k j}\right| \varphi\left(\left|Q_{k j}\right|\right)\left|Q_{k j}\right|} \int_{E_{k j}}\left(\int_{E_{k j}} f d \mu\right) d \mu\right) \chi_{Q_{k j}} \\
& \leq \frac{1}{1-\alpha} \sum_{k \in \mathbf{Z}, j \in \overline{I_{k}}}\left(\frac{1}{\left|Q_{k j}\right|} \int_{E_{k j}}\left(\sum_{m \in \mathbf{Z}, n \in \overline{I_{k}}} \frac{1}{\varphi\left(\left|Q_{m n}\right|\right)} \int_{E_{m n}} f d \mu\right) \chi_{E_{m n}} d \mu\right) \chi_{Q_{k j}} \\
& =\frac{1}{1-\alpha} T_{1}^{\prime} T_{2} f .
\end{aligned}
$$

Since $\left|T_{1} y\right| \leq M y$ and $M$ is a bounded operator from $X_{w}$ into $X_{w}, T_{1}^{\prime}$ is bounded in $\left(X_{w}\right)^{\prime}$. Thus, we obtain

$$
\left\|T_{0}^{\prime} f\right\|_{\left(X_{w}\right)^{\prime}} \leq(1-\alpha)^{-1}\left\|T_{1}\right\|_{X_{w} \rightarrow X_{w}}\left\|T_{2} f\right\|_{\left(X_{w}\right)^{\prime}}
$$

Hence $T_{0}^{\prime}$ is bounded whenever $T_{2}: X_{w} \rightarrow Y_{v}$ is bounded as well. But $T_{2}$ is the averaging operator defined by the sets $E_{k j}$. Similarly as in the proof of Theorem 2.1, one can prove that the operator $T_{2}$ is bounded provided $(w, v) \in A_{\varphi}(X, Y)$ and $(X, Y) \in \mathbf{G}$.

This completes the proof.

Remark. Let $t_{0} \in \mathbf{R}^{n}$ and $M_{\varphi}\left(k_{0}, t_{0}\right)$ be the Hardy-Littlewood maximal function, constructed on cubes $Q$ with sides $\leq 2^{k_{0}}$ and $Q-t_{0}\left(Q-t_{0}\right.$ is a translation $Q$ on $\left.t_{0}\right)$ dyadic cubes. Theorem 2.4 is true for the operator $M_{\varphi}\left(k_{0}, t_{0}\right)$. 
Now we remark that $M_{\varphi}\left(k_{0}, t_{0}\right) x \uparrow M_{\varphi}\left(\infty, t_{0}\right) x\left(k_{0} \uparrow \infty\right)$ and spaces $X$ and $Y$ are minimal. Then Theorem 2.4 is true for the operator $M_{\varphi}\left(\infty, t_{0}\right)$ and the norm of the operator $M_{\varphi}\left(\infty, t_{0}\right)$ does not depend on $t_{0}$. Here we use the monotone convergence theorem.

Let $M_{\varphi}$ be a Hardy-Littlewood maximal function, constructed on any cubes. Then we have $M_{\varphi} x \geq M_{\varphi}\left(\infty, t_{0}\right) x$ for all $x$.

We can use the arguments of [12, pp. 8-9]. Then we can say for $x \in X_{w}$ and $\varphi\left(2^{n} t\right) \leq C_{3} \varphi(t)\left(t \in \mathbf{R}_{+}\right)$that

$$
\left\|M_{\varphi} x\right\|_{Y_{v}} \leq C_{4} \sup _{t_{0} \in \mathbf{R}^{n}}\left\|M_{\varphi}\left(\infty, t_{0}\right) x\right\|_{Y_{v}}
$$

where $C_{4}$ does not depend on $x \in X_{w}$.

Therefore Theorem 2.4 is true for the Hardy-Littlewood maximal function in any cubes.

Example 1. Let $X=L_{p}$ and let $w$ be a weight satisfying the Muckenhoupt $A_{p^{-}}$ condition. If $Y$ satisfies an upper $q$-estimate with $q \geq p$, then the operator $M_{\varphi}$ is bounded from $\left(L_{p}\right)_{w}$ into $Y_{v}$ if and only if $(w, v) \in A_{\varphi}(X, Y)$.

Example 2. Let $X=L_{h_{0}}, Y=L_{h_{1}}$ be a couple of Orlicz spaces, constructed by $N$-functions $h_{0}, h_{1}$, and

$$
\sup _{1 \geq \lambda>0} \sup _{t>0} \frac{h_{0}^{-1}(\lambda t)}{h_{0}^{-1}(t)} \sup _{t>0} \frac{h_{1}^{-1}(t)}{h_{1}^{-1}(\lambda t)}<\infty .
$$

Using Theorem 20 in [1] we have $\left(L_{h_{0}}, L_{h_{1}}\right) \in \mathbf{G}$. Therefore the operator $M_{\varphi}$ is of weak type if and only if

$$
\sup _{Q} \frac{1}{\varphi(|Q|)}\left\|\chi_{Q} w^{-1}\right\|_{\left(L_{h_{0}}\right)^{\prime}}\left\|\chi_{Q} v\right\|_{L_{h_{1}}}<\infty .
$$

\section{REFERENCES}

1. E. I. Berezhnoi, Sharp estimates for operators on cones in ideal spaces, Trudy Mat. Inst. Steklov. 204 (1993), 3-36; English transl. in Proc. Steklov Inst. Math. 1994, no. 3 (204). MR 96a: 46052

2. H. M. Chung, R. A. Hunt and D. S. Kurtz, The Hardy-Littlewood maximal functions on $L(p, q)$ spaces with weights, Indiana Univ. Math. J. 31 (1982), 109-120. MR 83b:42021

3. E. M. Dynkin and B. P. Osilenkier, Weighted estimations of singular operators and their applications, Itogi Nauki i Tekhniki: Mat. Anal., vol. 21, VINITI, Moskow, 1983, pp. 42-130 (Russian); English transl. in J. Soviet Math. 30 (1985), no. 3.

4. J. Garcia-Cuerva and J. L. Rubio de Francia, Weighted norm inequalities and related topics, Math. Studies 116, North-Holland, Amsterdam, 1985. MR 87d:42023

5. Z. G. Gorgadze and V. I. Tarieladze, On geometry of Orlicz spaces, Lecture Notes in Math. 828 (1980), 47-51. MR 82h:46049

6. B. Jawerth, Weighted inequalities for maximal operators, Amer. J. of Math. 108 (1986), 361-414. MR 87f: 42048

7. V. M. Kokilashvili, The maximal functions and the integrals of the potential type in the Lebesgue and Lorentz spaces, Trudy MIAN SSSR 172 (1985), 192-201 (Russian).

8. J. Lindenstrauss and L. Tzafriri, Classical Banach spaces II, Function Spaces, Springer-Verlag, 1979. MR 81c: 46001

9. B. Muckenhoupt, Weighted norm inequalities for the Hardy maximal function, Trans. Amer. Math. Soc. 165 (1972), 207-227. MR 45:2461

10. S. Ya. Novikov, Cotype and type of Lorentz function spaces, 32, 2 (1982), 213-221; English transl. in Math. Notes 32 (1982). MR 84d:46031 
11. C. Perez, Two weighted norm inequalities for the Riesz potentials and uniform $L^{p}$-weighted Sobolev inequalities, Indiana Univ. Math. J. 39 (1990), 31-44.

12. E. T. Sawyer, A characterization for two weight norm inequalities for maximal operators, Studia Math. 75 (1982), 1-11.

Faculty of Mathematics, Yaroslavl State University, ul Sovietskaya 14, 150000 YAROSLAVL, RUSSIA

E-mail address: smirn@gw.yspu.yar.ru 